Norois

Environnement, aménagement, société

$230 \mid 2014$

Histoire de la géographie, mobilités étudiantes, circuits courts, maillage territorial, régénération urbaine, hydrographie

\title{
Régénération urbaine et développement territorial par le sport : Le cas du Parc olympique de Montréal
}

Urban regeneration and urban development through sport: The case of the

Olympic park in Montreal

Romain Roult, Sylvain Lefebvre et Jean-Marc Adjizian

\section{OpenEdition}

Édition électronique

URL : https://journals.openedition.org/norois/5020

DOI : 10.4000/norois. 5020

ISBN : 978-2-7535-3509-1

ISSN : $1760-8546$

Éditeur

Presses universitaires de Rennes

Édition imprimée

Date de publication : 30 juin 2014

Pagination : 57-72

ISBN : 978-2-7535-3464-3

ISSN : 0029-182X

\section{Référence électronique}

Romain Roult, Sylvain Lefebvre et Jean-Marc Adjizian, «Régénération urbaine et développement territorial par le sport : Le cas du Parc olympique de Montréal », Norois [En ligne], 230 | 2014, mis en ligne le 30 juin 2016, consulté le 13 janvier 2022. URL : http://journals.openedition.org/norois/5020 ; DOI : https://doi.org/10.4000/norois.5020 


\title{
Régénération urbaine et développement territorial par le sport : le cas du Parc olympique de Montréal
}

\author{
Urban Regeneration and Urban Development Through Sport: \\ The Case of the Olympic Park in Montreal
}

\author{
Romain Roult ${ }^{* a}$, Sylvain Lefebvre ${ }^{\mathrm{b}}$ et Jean-Marc AdjIZIAN ${ }^{\mathrm{c}}$
}

\begin{abstract}
*Auteur correspondant
a Ph. D. et professeur au Département d'études en loisir, culture et tourisme, Université du Québec à Trois-Rivières,

3351, boul. Des Forges, C.P. 500, Pavillon Ringuet (local 4030), Trois-Rivières, (Québec), G9A 5H7, Tel. 819-376-

5011 poste 3286, Fax.819-376-5158 (romain.roult@uqtr.ca)

b Ph. D. et professeur au Département de géographie de l'Université du Québec à Montréal

c Administrateur principal du Groupe de recherche sur les espaces festifs (www.gref.ca)
\end{abstract}

Résumé : Les Jeux Olympiques sont désormais largement utilisés à des fins de développement territorial. À ce titre, les infrastructures sportives construites à l'occasion de ces méga-événements s'insèrent pour certaines dans de vastes projets de régénération urbaine. C'est ce que cherchera à mettre en lumière cet article à travers une étude de cas sur le Parc olympique de Montréal. Par le biais de plusieurs analyses documentaires et de différentes entrevues, cette étude vise à distinguer les enjeux urbains et de gouvernance relatifs au développement sportif de ce site. Les résultats obtenus font émerger deux visions opposées au sujet du redéveloppement du Parc olympique de Montréal mais celles-ci s'accordent sur le fait que les deux principaux défis à surmonter relèvent de la justification et de l'origine des ressources financières et des modalités de gouvernance et de gestion à adopter et à appliquer.

\begin{abstract}
Olympic games are often a catalyst for urban development. The sport infrastructures and equipments constructed for these mega-events are sometimes integrated in wider urban regeneration strategies. This article will illustrate this phenomenon with the case study of Montreal and its Olympic site. Through a number of analysis, documents and interviews with sport agencies, local stakeholders and others, our study indicates the urban challenges and the governance issues related to the future of the Olympic Park. Results are showing two very different visions of redevelopment but with a consensus on two specific issues: the justification and the nature of major financial resources and the governance mix or «management model » needed for the upgrading of the site.
\end{abstract}

Mots clés : jeux olympiques - régénération urbaine - sport - Montréal - héritages - stade

Keywords: olympic games - urban regeneration - sport - Montreal - legacies - stadium

\section{INTRODUCTION}

La gestion des héritages olympiques est devenue une étape clé dans le processus de reconversion des villes hôtes des Jeux Olympiques (JO) (Smith, 2012). L'ampleur des investissements financiers nécessaires pour accueillir ces méga-événements 
et le nombre important d'infrastructures permanentes construites ou réaménagées à l'occasion de ces JO ont conduit ces dernières années les instances publiques ou privées impliquées dans ces projets urbains à considérer finement cette phase post-olympique (Pitts et Liao, 2009). Le Comité International Olympique (CIO) tente de sensibiliser depuis la fin des années 90 les villes candidates à l'organisation des $\mathrm{JO}$ de l'importance de planifier adéquatement l'après Jeux en prenant en compte notamment les besoins locaux et de fait en envisageant des équipements adaptés à ces réalités urbaines (Liao et Pitts, 2006). Même si plusieurs cas démontrent que ces recommandations ne sont pas toujours prises en compte (Athènes 2004 et Pékin 2008), il n'en reste pas moins que plusieurs villes olympiques comme Vancouver ou Londres ont tenté de planifier des Jeux dans une perspective de durabilité du legs olympique (Poynter, 2009). À ce titre, ces expériences métropolitaines ont montré qu'un des outils de développement le plus souvent utilisé est celui d'intégrer la construction ou le réaménagement d'équipements sportifs ou ludiques dans de vastes processus de régénération urbaine (Smith, 2012). Au-delà d'essayer d'intégrer ces infrastructures sportives aux paysages de ces villes, ces stratégies de régénération urbaine sont également souvent utilisées pour légitimer ces grands projets urbains auprès des populations locales (Gold et Gold, 2011).

Toutefois, cette question de la gestion des héritages olympiques n'est pas l'apanage de ces seules récentes villes olympiques. En effet, des métropoles ayant accueillies les JO dans les années 60, 70 et 80 sont encore aux prises avec des choix cruciaux au sujet du développement de leurs installations olympiques (Roult et Lefebvre, 2010). À ce titre Montréal, grâce notamment aux Jeux Olympiques de 1976, a réussi son pari de devenir une ville phare dans les domaines sportif et festif mais à un prix trois fois plus important que celui prévu initialement (400 millions \$ à plus de 1,2 milliards \$). Ce surcoût colossal contracté pour les JO et acquitté 30 ans après la fin de ces derniers a engendré d'importants problèmes de gestion et de reconversion de ces installations olympiques et a entaché la réputation de cette ville. Ces déboires financiers et de planification sont principalement à mettre au compte de politiques dirigistes menées par le maire de l'époque Jean Drapeau pour organiser ces JO (choix des sites des installations sans consultation publique ni études de besoins et nomination imposée de l'architecte en chef français Roger Taillibert) et à des contextes politiques et économiques assez moroses (crise souverainiste, inflation boursière et choc pétrolier) (Latouche, 2011). Depuis la fin des JO, la Régie des Installations Olympiques (RIO) est l'organisation publique responsable de la gestion et de l'exploitation du Parc olympique (figure 1). Toutefois, la RIO n’a jamais réussi à rendre ce site attractif malgré plusieurs stratégies urbaines, et ce en raison de la succession de problèmes techniques qui ont nui à l'image du stade ${ }^{1}$, par le départ de l'équipe professionnelle de baseball des Expos en 2004, mais surtout par la démesure d'un stade par rapport aux besoins réels de Montréal et par la nonattractivité d'un territoire d'accueil dépourvu de services susceptibles de combler la distance qu'il y a entre ce stade et le centre-ville.

Partant de ce portrait assez négatif, la RIO et le gouvernement provincial du Québec, a mandaté un comité-conseil en septembre 2011 visant à réfléchir à l'avenir du Parc olympique mais surtout à mener différentes consultations publiques auprès de 3805 personnes issues de la population québécoise et des milieux politiques, économiques et sociaux locaux (tableau 1). Plusieurs recommandations semblent faire consensus dans le cadre de ces consultations (Comité-conseil sur l'avenir du Parc Olympique, 2011). Tout d'abord, le Parc olympique apparaît comme un repère identitaire pour Montréal et pour l'ensemble du Québec et présente un énorme potentiel récréotouristique. Cependant ce potentiel n'est que très faiblement exploité et mis en valeur. Il semble également fondamental de mieux intégrer ce site à la trame urbaine environnante autour de logiques multifonctionnelles et de développement durable. Parallèlement, si le changement de la structure du toit du stade semble un préambule indispensable avant toute nouvelle opération de régénération urbaine de ce site, il apparaît également primordial de renouveler les stratégies de communication, publicitaires et de marketing

1. Plusieurs fissures et autres chutes de béton sur la structure du stade ont été recensées durant les années quatre-vingt et quatre-vingt-dix. Depuis 1987 et le choix d'installer un toit fixe sur le stade, plusieurs déchirures ont eu lieu dont la plus importante en 1999 qui a conduit à la fermeture de la surface de jeu de novembre à mars de chaque année, et ce encore aujourd'hui. 
dans l'optique de refonder l'image du Parc olympique et de le repositionner au niveau touristique. Finalement, même si de récentes activités culturelles, scientifiques et patrimoniales ${ }^{2}$ sont venues se greffer à ce Parc olympique, ces consultations publiques ont clairement démontré que le sport, dans ses nombreuses manifestations, doit rester un élément central dans l'orientation de développement du site mais à travers de nouvelles formes de gouvernance davantage partenariales.

Ce dernier constat, ressorti durant ce processus de consultations publiques, a été l'élément initiateur de cette recherche. En effet, nous avons tenté de comprendre comment les acteurs publics, privés et parapublics agissant de près ou de loin sur le Parc olympique perçoivent les activités sportives offertes et de quelles manières ils envisagent le développement de ce site sous l'angle du sport, et ce tant au niveau de la programmation événementielle qu'au sujet de la gouvernance.

\section{JEUX OLyMPIQUES, RÉGÉNÉRATION URBAINE ET DÉVELOPPEMENT TERRITORIAL}

\section{Les projets olympiques comme stratégies de régénération urbaine : Fabrication et transformation de la ville par le sport}

Se manifestant depuis plus de cinq décennies dans les pays occidentaux, la régénération urbaine renvoie désormais à un urbanisme de transformation et non d'expansion, caractérisé par un retour à la ville d'un point de vue surtout qualitatif et à un renforcement de la centralité urbaine (PAP, 2004). Il faut donc comprendre cette notion comme un nouveau mode de fabrication et de transformation de la ville (Tallon, 2010). Dans ce contexte, les méga-évènements sportifs et culturels ont pris une importance accrue et occupent désormais une place centrale dans les plans de développement et de régénération des métropoles contemporaines. Smith (2012) mentionne notamment que : «Événement et régénération sont des concepts qui se retrouvent dans des lieux spécifiques, mais sont également deux importants mécanismes de transformation et

2. On pense bien entendu à ce titre au complexe muséal de nature dénommé « Espace pour la vie Montréal » qui regroupe le biodôme, le planétarium,

l'insectarium et le jardin botanique de Montréal.

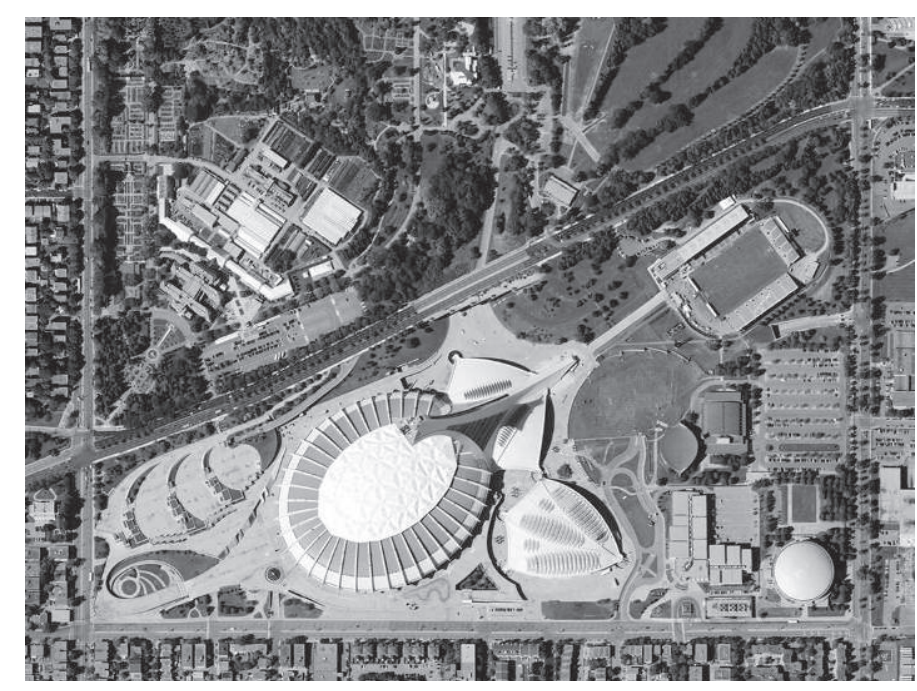

Figure 1: Photo aérienne du Parc olympique de Montréal - Source : Google Map Aerial view of the Montreal Olympic Park

\begin{tabular}{|c|c|}
\hline \multicolumn{2}{|c|}{ Participation aux activités de consultation de l'automne 2011} \\
\hline Activités & Nombre de personnes \\
\hline $\begin{array}{l}\text { Visiteurs uniques sur le site } \\
\text { internet }\end{array}$ & 2998 \\
\hline $\begin{array}{l}\text { Visiteurs des installations } \\
\text { olympiques }\end{array}$ & 651 \\
\hline $\begin{array}{l}\text { Consultations ciblées à } \\
\text { Montréal }\end{array}$ & 112 \\
\hline Consultations en région & 44 \\
\hline Total & 3805 \\
\hline \multicolumn{2}{|c|}{ Consultations en région en octobre 2011} \\
\hline Région & Représentants d'organismes \\
\hline Rimouski & 14 \\
\hline Rouyn-Noranda & 10 \\
\hline Sherbrooke & 6 \\
\hline Mont-Laurier & 14 \\
\hline Total & 44 \\
\hline \multicolumn{2}{|c|}{ Secteurs d'activités des organisations consultées } \\
\hline Secteurs & Nombre d'organisations \\
\hline Tourisme & 13 \\
\hline Art & 13 \\
\hline Éducation & 8 \\
\hline Affaires & 15 \\
\hline Patrimoine & 18 \\
\hline Communautaire & 12 \\
\hline Sport & 18 \\
\hline Riverains & 15 \\
\hline Total & 112 \\
\hline
\end{tabular}

Tableau 1: Volets des consultations publiques menées par le Comitéconseil sur l'avenir du Parc olympique (Source : Comité-conseil sur l'avenir du Parc Olympique [2011])

Public consultations conducted by the advisory committee on the future of the Montreal Olympic Park 
de développement des espaces. Ces deux notions sont de véritables agents de territorialisation. » (2012 : 10. Traduction libre ${ }^{3}$.) Cet auteur, tout comme Tallon (2010), vont même encore plus loin dans l'interprétation conceptuelle de cette relation entre les notions d'événement et de régénération. Pour eux, il existe deux principales formes de régénération urbaine via des événements conditionnées par l'utilisation faite par les élites politiques et économiques de ces manifestations. La première forme, qualifiée de « event-led regeneration » ou « régénération basée sur l'événement », où « le processus de changement est entraîné par l'événement » (Smith, 2012 : 11. Traduction libre) $)^{4}$. Diverses infrastructures sportives, culturelles et d'accueil sont donc construites ou modernisées à cet effet puis adaptées pour leur reconversion post-événementielle. La seconde forme, dénommée « event-themed regeneration » ou « régénération liée à la thématique événementielle », se réfère à une stratégie de planification plus large où les objectifs urbains fixés dépassent l'organisation même de l'événement (Smith, 2012; Tallon, 2010). Cette manifestation est donc vue ou utilisée comme levier de développement permettant d'attirer un plus grand nombre d'investisseurs et parallèlement de diminuer les temps de réalisation de ces projets urbains. À ce titre, les Jeux Olympiques d'été se placent notamment comme un élément constituant ce système où par le biais de différentes réalisations urbaines, qu'elles soient bâties ou naturelles, cette manifestation devient un vecteur à moyen et long termes de régénération de la ville ou du moins de certaines parties (Shirai, 2009).

Ces dernières années, la relation entre le sport et les stratégies de régénération urbaine s'est largement développée au sein des appareils politiques locaux et nationaux (Hay et Cashman, 2008). Cela est principalement lié aux avantages économiques et sociaux perçus et associés à l'organisation de méga-événements festifs et au développement des équipements d'accueil connexes. Ainsi ces nouvelles stratégies urbaines, liant le sport et l'événementiel à la ville, ont conduit indéniablement à l'élaboration de nouveaux programmes politiques et d'aménagement du territoire dans lesquels des mesures de reconversion

3. Citation originelle : "Events and regeneration are concepts which occur in specific places, but they are also both important mechanisms through which places are made. They are both agents of territorialisation."

4. Citation originelle: "The process of change is driven by the event. " économique et de revitalisation urbaine et durable sont de plus en plus présentes (Smith, 2012). Pitts et Liao (2009) indiquent que les impacts et autres transformations des JO sur les territoires hôtes se reflètent à différents niveaux géographiques :

- à l'échelle régionale, nationale et internationale où on observe des répercussions sur le réchauffement climatique et l'épuisement de diverses ressources naturelles;

- à l'échelle urbaine, il est possible de constater que les $\mathrm{JO}$ peuvent conduire à une restructuration de la forme urbaine, à une amélioration des systèmes de transport privés et publics, et à une réorganisation des fonctionnalités des quartiers centraux et périphériques et des densités urbaines;

- à l'échelle locale, ces méga-événements peuvent transformer la forme physique de divers quartiers (grille de rues, forme des façades, configuration des espaces verts, etc.), mais également modifier les ambiances et autres logiques commerciales de ces territoires.

Parallèlement, Pitts et Liao ajoutent que : «L'idée du "Mouvement Olympique moderne" n'est pas fondamentalement un concept géographique et la notion de "ville olympique" n'est pas une conception urbaine spécifique, mais ils sont plutôt des phénomènes flexibles constamment enrichis et réinterprétés par les nouvelles planifications urbaines suivies par les villes hôtes et par l'organisation des plus récents Jeux Olympiques. » (2009: 46. Traduction libre ${ }^{5}$.)

\section{Public ou privé? La question de la gouvernance urbaine dans la planification des Jeux Olympiques}

En préambule à cette sous-partie, il nous apparaît important de rappeler la place désormais prégnante de l'approche par instruments dans la gouvernance territoriale. Thévoz (2013) mentionne à ce titre que : « les pouvoirs publics ont un besoin croissant - en tant que détenteurs du pouvoir public légitime d'instruments pour organiser de manière productive des délibérations d'un grand nombre d'acteurs appelés à donner un contenu (nouveau) à la gouvernance

\footnotetext{
5. Citation originelle : "The idea of a 'Modern Olympia' is not a truly geographic concept and the 'Olympic City' is not a distinct urban genre; rather, they are flexible phenomena constantly enriched by the practice of host cities and reinterpreted through the preparation for each new Games. »
} 
territoriale» (2013: 231). Pour Lascoumes et Le Galès (2007), chaque phase de développement ou de restructuration de l'État central est accompagnée par une nouvelle vague d'innovation en matière d'instruments de planification et de production de l'espace urbain. Pour ces auteurs, l'augmentation du nombre d'acteurs investis dans les projets urbains contemporains explique le besoin fécond d'instruments politiques et consultatifs visant notamment à faciliter ces échanges et à répondre à divers objectifs de coordination. À ce titre, Vodoz et al. (2013) mentionnent que la gouvernance territoriale est dorénavant une science de l'aménagement fondée sur la recherche d'un équilibre entre des intérêts particuliers (monoscalaires et/ou sectoriels), une cohérence de l'action publique et certaines garanties démocratiques. De son côté, Mévellec (2006), en se basant sur les travaux de Lascoumes et Le Galès, estime que trois leçons se dégagent de cette gouvernance par instruments, en l'occurrence que : « le changement d'instrument représente souvent l'invalidation des instruments précédents plutôt qu'une modification des objectifs de l'action publique; travailler sur et à partir des instruments permet de mieux évaluer les transformations réelles de l'action publique; les instruments ne sont pas des techniques neutres mais participent à la production et à la stabilisation de représentations sociales et politiques qui, elles-mêmes, structurent l'espace politique» (2006: 446).

Dans la perspective et l'histoire des Jeux Olympiques, la planification de l'organisation de ces méga-événements dans une perspective de régénération urbaine a été effectuée à travers trois principaux modèles de gouvernance (Poynter, 2009). Le premier est fondé sur une structure de gestion uniquement publique où les gouvernements nationaux et provinciaux jouent des rôles clés au niveau notamment du financement des principales infrastructures. Le second modèle, qualifié de «modèle partenarial public-privé », se base sur un fonctionnement décisionnel où des acteurs publics et privés, théoriquement à part égale, déterminent ensemble une vision et des objectifs de planification des JO. Ce modèle conduit donc en principe à une gestion de risques partagée des projets urbains mis en chantier. Finalement le troisième modèle se réfère à une structure de gouvernance par « réseaux d'acteurs » où différents intérêts émanant de divers milieux (État central, province, ville, secteur privé, mouve- ments associatifs, groupes de citoyens, etc.) touchés de près ou de loin par ces projets olympiques sont débattus et permettent l'élaboration d'une vision commune d'action.

Ces trois modèles théoriques sont toutefois difficiles à distinguer clairement dans la pratique ou peuvent dans certains cas changer de formes durant le processus de planification des JO (Gold et Gold, 2011). Comme l'indique Poynter (2009): «Les structures de gouvernance peuvent prendre la forme de réseaux fondés sur diverses parties prenantes mais dans ce type de développement de projet différents interêts commerciaux peuvent prévaloir (Atlanta 1996) ou bien l'équilibre proposé entre les financements publics et privés peut être compromis en raison de conditions économiques externes qui empêchent ces acteurs privés à engager les capitaux suffisants pour réaliser le projet de la manière initialement prévue (Londres 2012).»(2009: 16. Traduction libre ${ }^{6}$.) Smith (2012) indique que la présence de plus en plus accrue du secteur privé dans ces projets olympiques dans une perspective de régénération se remarque depuis le début des années 90 et se réfère aux modèles progressistes néo-libéralistes de développement urbain. Cet auteur ajoute que : «C'est pourquoi nous parlons maintenant d'arrangements de gouvernance ainsi que de la responsabilité du gouvernement lors de débats sur des politiques de régénération » (Smith, 2012: 223. Traduction libre) ${ }^{7}$. Smith (2012), tout comme Orueta et Fainstein (2008), mentionnent cependant que quel que soit le type de partenariats retenu, exclusif (public ou public-privé) ou inclusif (réseaux d'acteurs), le principe de base au niveau de la gouvernance est presque toujours le même, soit que le secteur public initie le projet en faisant l'acquisition de terrains constructibles par exemple et en déléguant par la suite la construction et la gestion des équipements nécessaires aux acteurs privés et parapublics. Cette implication moins conséquente de ces partenaires non-gouverne-

\footnotetext{
6. Citation originelle : "Governance frameworks may take the form of a network of stakeholders but in the process of a project's development, specific commercial interests may prevail (Atlanta 1996) or the proposed balance of private/public funding may be undermined by external economic conditions that impede the private sector's capacity to raise sufficient capital to fully engage with the project in the manner intended (London 2012). »

7. Citation originelle: "That is why we now talk about governance arrangements as well as government responsibility when discussing regeneration policy. »
} 
mentaux et davantage fondée sur des logiques de sous-traitance augmente automatiquement le risque que ces acteurs disparaissent ou diminuent leurs implications lorsque le climat économique ambiant devient morose (Gold et Gold, 2011). Dans ce cas, les seuls risques encourus sont donc pris par le secteur public. Ces constations conduisent Davies (2002) à parler de « mariages de complaisance » pour qualifier ces différentes formes de partenariats. Pour cet auteur mais également pour Dargan (2009), ces modèles de gouvernance incluant plusieurs types d'acteurs ne sont souvent que des ententes de "façade » où seuls les intérêts des organisations les plus puissantes primeront. Orueta et Fainstein (2008) vont même plus loin en indiquant que ces nouvelles formes de gouvernance partenariales ne font que répéter les erreurs du passé en mettant uniquement de l'avant les intérêts économiques au détriment des préoccupations locales et citoyennes.

Ces modèles de gouvernance incluent à la base la planification de la phase post-olympique mais plusieurs auteurs constatent cependant que ces stratégies de gestion ont tendance à changer de formes lorsque les JO se terminent (Smith, 2012; Poynter, 2009). En effet, ces dernières, et en particulier celles concernant l'exploitation du Parc olympique, sont le plus souvent menées par une organisation publique qui a pour seule fonction de gérer le développement de ce site (Poynter, 2009). Toutefois, certaines de ces agences publiques choisissent de concéder l'exploitation de diverses infrastructures à des entreprises privées de manière à minimiser temporairement les risques reliés à la mise en marché de ces équipements (Hay et Cashman, 2008). Ces ententes peuvent pour certains permettre de dynamiser le développement de ces tissus urbains mais pour d'autres sont en fait de simples «trompes l'œil» puisque seules les installations les plus rentables seront retenues et donc exploitées par les acteurs privés (Gibbons et Wolff, 2012; Glynn, 2008). Le reste du "fardeau structurel » olympique se révèle donc à la seule charge du secteur public. Poynter (2009) stipule à ce titre que : «l'équilibre entre « le commercialement viable » et le «socialement désirable » au sujet de la gestion des héritages olympiques est une problématique qui a fait l'objet d'importants débats durant les phases post- olympiques de plusieurs villes hôtes » (2009: 37. Traduction libre) $)^{8}$.

\section{Développement territorial et Jeux Olympiques : un pari risqué qui doit de plus en plus se calquer sur les réalités locales}

Miser sur des méga-événements, en particulier sur les JO, pour développer certains territoires est une opération risquée et semer « d'embûches » pour plusieurs auteurs (Westerbeek, 2009; Flyvbjerg et al., 2003). Le premier et souvent le principal danger potentiel est l'écart existant entre les exigences des Fédérations Internationales et autres organisations mondiales sportives pour l'accueil de ces manifestations et les besoins locaux en matière de sport. Ce risque est bien entendu encore plus prégnant lorsque l'on parle d'importants équipements sportifs, tels un stade, un amphithéâtre ou encore un centre aquatique. Pour Smith (2012), il est assez facile de tomber dans l'euphorie des JO en planifiant des équipements grandioses et spectaculaires mais toutefois complétement déconnectés des réalités locales. Pour cet auteur tout comme pour Silk (2002), cela multiplie les risques que ces infrastructures deviennent des "éléphants blancs ». À cet effet, Smith (2012) précise que : «Il est de plus en plus convenu que de nouvelles installations doivent être construites uniquement s'il existe un besoin en la matière. Si ce n'est pas le cas, d'autres infrastructures existantes, des éléments temporaires ou encore des installations localisées dans d'autres villes limitrophes doivent être utilisés. » (2012 : 68. Traduction libre $)^{9}$. L'intégration de ces grands projets structurels dans un processus de régénération urbaine tend à minimiser ces risques, et ce en raison de la nature même de ces stratégies de développement. Toutefois dans ces cas, le défi résulte davantage dans la capacité à aller chercher des partenaires financiers et parallèlement l'appui de la population. Selon Smith (2012), la présence et l'implication de «forces externes », autres que l'État central, la province, la ville-centre et le comité organisateur, sont

\footnotetext{
8. Citation originelle: "The balance of legacy development between the "commercially viable" and the 'socially desirable' has been the subject of considerable debate in host cities after the games/event. "

9. Citation originelle: «It is increasingly acknowledged that new venues should be developed only where there is a proven need for them; otherwise existing venues, temporary venues or facilities in nearby towns should be employed. »
} 
essentielles pour assurer une planification adaptée aux milieux hôtes de ces méga-événements. De son côté Poynter (2009) stipule que l'enjeu central dans ces stratégies de développement territorial est relié à l'acquisition du foncier.

À ce titre, la présence unique de pouvoirs publics dans ces grands projets de régénération urbaine est souvent suivie mais demande à ces acteurs d'être capables de justifier et de légitimer ces investissements majeurs aux populations locales. Ceci est principalement effectué en minimisant les coûts de planification et de construction de ces stratégies de développement. L'autre solution envisageable est celle de léguer la gestion et l'exploitation du site au secteur privé. Ces logiques sont cependant largement décriées par les communautés résidentes notamment puisque ces acteurs privés profitent d'équipements construits par le secteur public en privatisant leurs accès. Zhang et Wu (2008) vont quant à eux parler de stratégies fondées sur des coalitions d'acteurs. Chaque infrastructure et autre espace constituant ces projets de régénération urbaine est évalué à la vue de son utilisation post-événementielle et permet de fait d'estimer les formes d'exploitation les plus viables à son encontre (publique, publique-privée et uniquement privée). Ces principes rejoignent la théorie énoncée et illustrée par Raco (2005) sur les cas de plusieurs villes britanniques. En effet, pour cet auteur, l'accueil de manifestations sportives peut être envisagé dans une optique de développement territorial mais se doit d'être mené par des organisations multipartites de développement urbain (public, privé et société civile). À ce sujet Smith indique que : « Les sociétés de développement urbain sont utilisées par les gouvernements pour développer des sites d'événements, assister la transformation de ces sites après l'événement et veiller à ce que ces processus de régénération s'étendent aux zones limitrophes du lieu de l'événement. »(2012: 242. Traduction libre ${ }^{10}$.) Cependant pour Majoor (2008), ce fonctionnement décisionnel multisectoriel doit être fondé sur une planification urbaine modulable et adaptable aux contextes économiques et sociaux locaux, et ce avant l'accueil du méga-événement concerné mais surtout après cette manifestation. Pour cet auteur,

10. Citation originelle : «Urban development corporations are used by governments to develop event sites, assist post-event transformation of those sites and/or to ensure that regeneration extends to areas beyond event venues. "
« Il est possible de connecter un programme de planification par étapes à un projet de développement à grande échelle avec un motif économique clair. Toutefois, si un tel programme n'est pas lié à des concepts et des outils de planification adéquats, son exécution risque d'être difficilement réalisable. » (Majoor, 2008 : 115. Traduction libre) ${ }^{11}$.

\section{Méthodologie}

D’un point méthodologique, cette recherche se base sur une démarche qualitative et une approche inductive et interprétative pour laquelle les techniques de collecte de données, de l'analyse de contenu et d'entrevues semi-dirigées, ont été utilisées. Cette stratégie méthodologique s'appuie sur deux phases.

Dans un premier temps, nous avons analysé de façon exhaustive l'ensemble des mémoires soumis au Comité-conseil sur l'avenir du Parc Olympique et déposés par des organisations gravitant dans le domaine du sport ${ }^{12}$. Toutefois, il est à noter que nous avons également consulté les autres mémoires présentés dans lesquels certains éléments évoqués relevaient du domaine sportif. Ainsi plus d'une quinzaine de mémoires ont été étudiés ${ }^{13}$. Au-delà d'une meilleure compréhension des intérêts, des préoccupations et des attentes de divers acteurs du monde du sport autour du développement du Parc olympique de Montréal, ces analyses de contenu nous ont également permis de cibler plusieurs organisations incontournables susceptibles d'être interviewées durant la seconde phase de notre collecte de données. De plus, ces mémoires ainsi que l'analyse du rapport-synthèse du Comité-conseil sur l'avenir du Parc Olympique (2011) nous ont aussi aidés à concevoir notre guide d'entretien.

Dans un second temps, 36 entrevues auprès de 21 organisations ont été menées durant l'année $2012^{14}$. Ces acteurs interviewés sont principale-

11. Citation originelle: «It is certainly possible to connect a progressive planning agenda to a large scale development project with a clear economic motive. However if such an agenda is not linked to adequate planning concepts and tools its execution will be disappointing. "

12. Il est à noter que ces différents mémoires ont été conservés en versions papier et électronique dans les archives de la Régie des Installations Olympiques et sont disponibles au grand public sur demande.

13. Cette analyse documentaire a été effectuée manuellement par recoupant thématiques selon les diverses problématiques sportives de notre étude.

14. La méthode d'échantillonnage retenue a été celle par choix raisonné. 23 organisations répondant à nos critères d'inclusion relatifs à l'implication dans le développement du sport sur le Parc olympique (compétitions, événements, gestion, etc.) ont été visées et contactées. 21 d'entreelles ont accepté de participer à l'étude. 
ment issus des milieux du sport-spectacle, du sport d'excellence, du sport amateur, du sport étudiant et scolaire, du monde associatif et public ainsi que du secteur privé (figure 2). Ces entrevues, organisées en face à face ou par téléphone ont permis de comprendre comment ces acteurs utilisent et gèrent différents plateaux sportifs présents sur le site et parallèlement, perçoivent et envisagent le développement de la vocation sportive du Parc olympique de Montréal. Six principaux thèmes ont été abordés durant chaque entrevue :

- les créneaux sportifs à conserver et à développer (sport-spectacle, sport d'excellence, sport amateur et sport à portée locale);

- les dimensions urbanistiques du Parc olympique liées au domaine sportif (aménagement et rénovation des plateaux sportifs, valorisation des espaces extérieurs, modernisation des commodités d'accueil, etc.);
- les dimensions touristiques du Parc olympique liées au domaine sportif (adaptation de la vocation sportive aux clientèles touristiques locales, nationales et internationales, mise en marché de l'offre touristique sportive, etc.);

- les dimensions de gouvernance du Parc olympique liées au domaine sportif (stratégies de gouvernance à renforcer ou à intégrer pour développer certains créneaux sportifs, mesures de légitimation de certains investissements auprès des populations locales, logiques partenariales lors de l'organisation de certains événements sportifs, etc.);

- Relations à développer avec les différentes organisations sportives ou non présentes sur le Parc olympique;

- Réactions par rapport à la synthèse des consultations publiques menées par le Comité-conseil sur l'avenir du Parc Olympique.

Plus spécifiquement, ces entretiens ont permis de cerner certains facteurs freinant ou limitant l'utilisa-

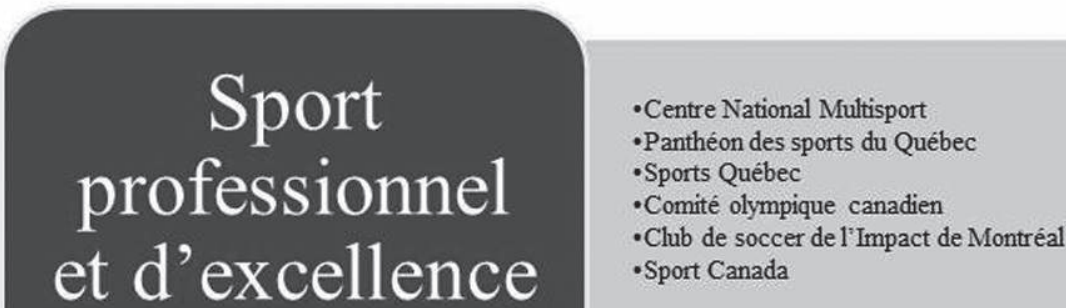

\section{Sport amateur et scolaire}

- Réseau du sport étudiant du Québec - Commission scolaire de Montréal -Défi sportif - Centre sportif du Parc olympique - Jeux de Montréal - Club de natation des piscines du Parc olympique
Milieux

associatif, public et privé
- Chambre de Commerce de l'Est de Montréal

- Conseil québécois du loisir

- Ville de Montréal et arrondissements

- Ministère del'Éducation, du Loisir et du Sport du Québec

- Membres du Comité de réflexion sur l'avenir du Parc olympique

-Muséums Nature Montréal

- Régie des Installations Olympiques

- Regroupement loisir et sport du Québec

-Québec en Forme

Figure 2: Catégorisation des organisations interviewées (Source : auteurs)

Types of organizations interviewed 
tion de divers plateaux sportifs. Concernant le traitement des données issues des entrevues semi-dirigées, elles ont été analysées via le logiciel NVivo ${ }^{15}$. Au-delà de la construction d'un arbre de codification, ce traitement informatique a permis de regrouper et de catégoriser les principaux résultats dans l'optique de dresser un portrait global des données obtenues par types de répondants et par thèmes analytiques. Il est à noter que la première phase de traitement de ces données qualitatives (regroupement et catégorisation) a été menée au départ par deux chercheurs de façon distincte. Chaque chercheur établissait lui-même sa propre analyse sans échanger avec l'autre chercheur. Puis une mise en commun était effectuée. Cette technique d'analyse a permis de minimiser les biais interprétatifs et ainsi renforcent l'objectivité des résultats présentés.

\section{Principaux résultats de RECHERCHE : UNE VOCATION SPOR- TIVE À PRÉSERVER ET À DÉVELOPPER}

Les consultations publiques sur l'avenir du Parc olympique tenues à l'automne 2011 ont clairement fait ressortir un consensus sur la nécessité de consolider et mettre en valeur la vocation sportive du site dans son ensemble. De façon naturelle, les intervenants consultés, les mémoires déposés et la population en général se rallient au fait que le sport est déjà la vocation fondatrice du site et que l'héritage des Jeux olympiques de 1976 mérite d'être remis en valeur pour refonder un sentiment de fierté sur le site. En complémentarité avec les fonctions culturelles, scientifiques et commerciales existantes, cette vocation sportive est déjà présente mais demeure sous-exploitée selon plusieurs documents analysés. À ce titre, nos résultats et les analyses corrélées nous ont permis de faire ressortir plusieurs éléments prioritaires faisant consensus auprès des acteurs interviewés (figure 3). Par ailleurs et selon les catégories d'organisations sollicitées, il nous a été possible de mettre en lumière d'autres paramètres importants pour ces dernières au sujet du dévelop-

15. Les transcriptions de l'ensemble des entrevues effectuées représentaient une somme très conséquente d'informations textuelles. De manière à faciliter l'analyse de ces discours, l'utilisation du logiciel NVivo nous offrait la possibilité d'effectuer rapidement et de façon systématisée la codification et l'organisation par types de répondants et par thèmes des données obtenues. pement de la vocation sportive du Parc olympique (figure 4).

Ce consensus sur le sport comme vocation principale du Parc olympique n'est pas une surprise car l'identité sportive des lieux est encore très présente sur un plan fonctionnel et sur un plan symbolique. À ce titre, un représentant d'une fédération sportive provinciale indique :

«Sur le plan fonctionnel, on retrouve des événements sportifs qui sont organisés de façon occasionnelle dans l'enceinte du stade et plusieurs équipements connexes fonctionnent pratiquement à pleine capacité (centre sportif et complexe aquatique notamment). Il y a donc une réelle volonté de préserver ce qui n’a pas été encore démantelé des équipements olympiques originaux. Si le vélodrome, la piste d'athlétisme et certains espaces intérieurs du stade ont été modifiés, il reste encore plusieurs lieux qui ne demandent pas mieux qu'un réaménagement ou une mise à niveau pour accommoder les nombreux besoins et multiples demandes des acteurs du sport ».

Le maintien de l'héritage olympique des Jeux de 1976, voire sa mise en valeur de façon plus dynamique, semble être importante auprès d'un grand nombre d'intervenants consultés. À ce titre, un membre du Comité Olympique Canadien stipule : "Historiquement, il n'y a aucune ville olympique qui ait renoncé à l'héritage laissé par ses jeux mais le maintien de ces héritages varie beaucoup d'une ville olympique à l'autre. Être membre du club très sélect des villes olympiques demeure encore une carte de visite et un vecteur de visibilité et de rayonnement international considérable. » Toutefois peu d'acteurs interpellés par cette problématique sont convaincus de la pertinence de musées des sports ou autres de ce genre. Ces derniers recommandent plutôt la mise en place de petites interventions ponctuelles, temporaires, innovatrices et interactives sur plusieurs endroits du site. Une commémoration expérientielle de ce legs olympique est le plus souvent privilégiée par ces acteurs, c'est-à-dire une expérience basée sur des technologies avec un récit dynamique dans l'espace, innovateur, de bon goût, etc. Seules quelques personnes interviewées suggèrent des formes de mise en valeur plus statiques de ce patrimoine olympique. Pour celles-ci, un espace spécifique pourrait ainsi être dédié à une exposition permanente sur les 


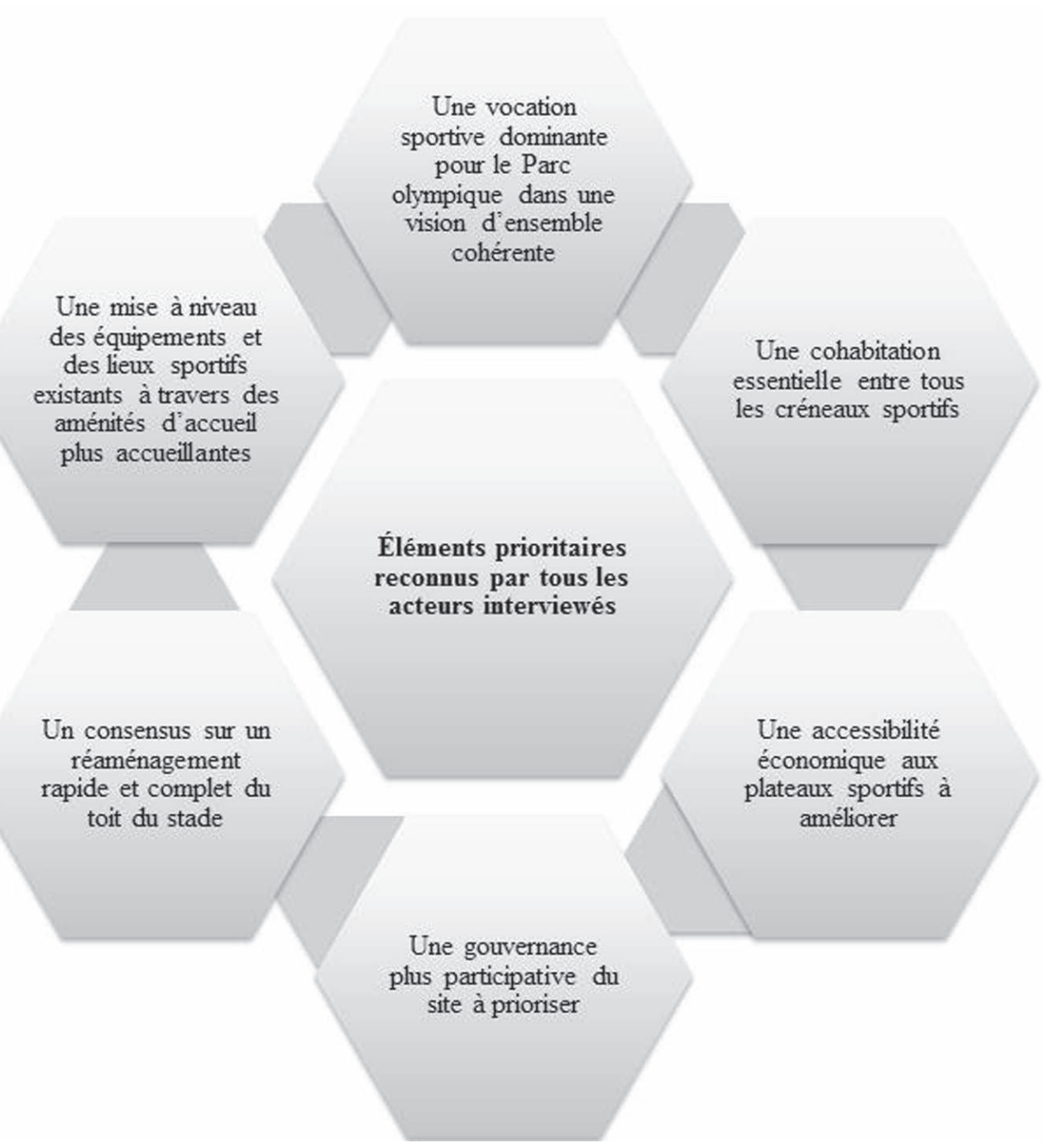

Figure 3 : Éléments prioritaires pour le développement de la vocation sportive du Parc olympique faisant consensus auprès des acteurs interviewés (Source : auteurs)

Priority elements for the stakeholders interviewed about the sport development of Montreal Olympic Park

Jeux Olympiques de 1976 et sur l'histoire du sport au Québec. Cet espace devrait, selon ces acteurs, être localisé dans le stade lui-même.

De façon globale, le sport-spectacle à grand déploiement (événements et championnats spéciaux, sport professionnel, matchs d'exhibition, etc.), le sport d'élite (d'excellence, olympique, haute performance) et le sport de portée locale (pratique libre, sport scolaire, sport amateur en général, etc.) sont les trois grandes catégories ou créneaux sportifs qui devraient être mis en valeur sur le site. La totalité des intervenants souhaite le maintien de ces trois créneaux mais parmi ceux-ci, une majorité aimerait voir une certaine priorité donnée au sport d'élite et de haute performance. Le centre sportif et les activités aquatiques répondent déjà partiellement à ce souhait et la venue prochaine de l'Institut National du Sport du Québec (INSQ) permettra, selon plusieurs, de consolider et de répondre à certains besoins des athlètes de haut niveau. À ce sujet, un représentant d'une organisation sportive fédérale stipule que : "Dans le respect de l'héritage olympique et avec le souci de répondre prioritairement aux besoins de l'excellence sportive à une échelle nationale avec des équipements de haut niveau, une priorisation de certains sports d'élite permettrait aussi un positionnement stratégique de Montréal face à d'autres villes canadiennes qui ont su capitali- 


\section{Sport professionnel et d'excellence}

- Le secteur privé a sa place sur le Parc olympique, à la fois via des activités et des services permettant d'animer et de dynamiser ce territoire mais aussi pour augmenter la rentabilité globale des actifs sur le site.

- Le sport d'excellence devrait être prioritaire sur les autres créneaux d'activités sportives.

- Les événements majeurs organisés doivent être uniques et originaux et se distinguer d'autres manifestations présentées ailleurs dans l'agglomération montréalaise.

\section{Sport amateur et scolaire}

- Promouvoir les saines habitudes de vie et la santé par le sport peut être une bonne façon de justifier les investissements massifs qui seront requis.

- Les milieux scolaires devraient être davantage intégrés aux activités sportives d'élite, professionnelle ou de spectacle via des partenariats éducatifs-participatifs.

- L'utilisation des espaces intérieurs de la Tour du stade devrait être renforcée via

l'aménagement de nouveaux plateaux sportifs.

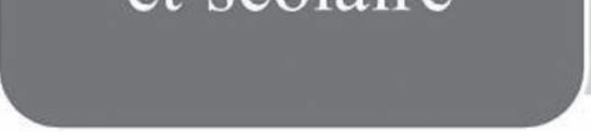

\section{Milieux \\ associatif, public et privé}

- Création d'un guichet unique pour les intervenants qui aimeraient organiser ou utiliser des équipements du Parc olympique.

- Les stratégies de marketing et de communication de la RIO devraient être renouvelées, plus ciblées voire même plus agressives.

- La valorisation de l'héritage olympique devrait être effectuée via des interventions muséales ponctuelles et temporaires.

Figure 4 : Catégorisation de certaines considérations de développement importantes pour les différents types d'organisations sollicitées Significant elements for different types of organizations interviewed about the development of Montreal Olympic Park

ser sur leurs équipements sportifs pour maintenir les athlètes dans leur marché métropolitain (Toronto, Vancouver, Calgary).»

Malgré une certaine priorisation du sport d'élite dans la consolidation de la vocation sportive du Parc olympique, il y a une nette volonté d'en faire profiter le sport amateur, le sport scolaire et de divertissement (figure 5). Pour une personne travaillant dans le domaine du sport scolaire :

« La présence des athlètes de haut niveau permettrait de faire des liens et des passerelles avec les autres créneaux sportifs par l'organisation d'activités spéciales et de journées découvertes et d'initiation auprès des écoles, des camps de jour ou camps d'été, des journées "portes-ouvertes" et thématiques avec échanges et interactions avec la population locale, métropolitaine voire même touristique. »
Par ailleurs, une des principales difficultés relevée par les intervenants interviewés concerne le problème d'accessibilité à certains espaces sportifs du Parc olympique. Pour un représentant d'une Fédération sportive provinciale: "C'est davantage le stade qui est concerné ici puisque le centre sportif fonctionne déjà à pleine capacité. Devant les coûts prohibitifs pour la location de certains espaces, plusieurs événements, championnats et autres activités ne peuvent être tenues dans le stade ou ailleurs. » De même, certaines contraintes administratives inhérentes au fonctionnement de la RIO (coûts d'entretien, syndicats, etc.) empêchent, selon plusieurs personnes interviewées, l'utilisation de bénévoles des fédérations sportives ou des établissements scolaires lors de la tenue de certaines activités. En somme, si on résume l'ensemble des commentaires et des analyses qui nous ont été proposés, le pro- 


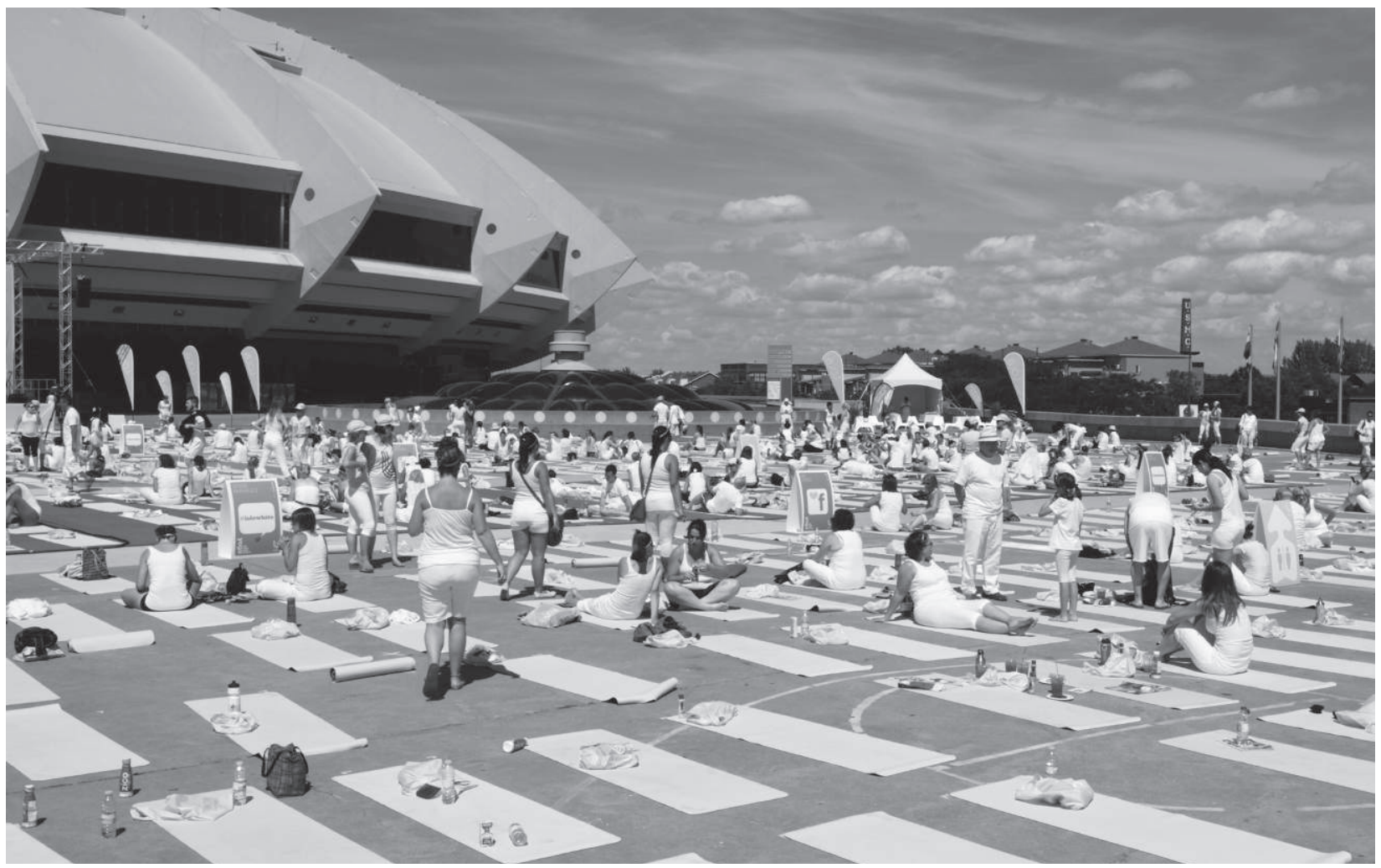

Figure 5 : Activité « Yoga en blanc » organisée sur l'esplanade Financière Sun Life du Parc olympique (Source : Sylvain Lefebvre [juillet 2013]) "White Yoga » event organized on the Sun Life Financial esplanade of the Montreal Olympic Park

blème prioritaire de la mise en valeur du sport sur le site du Parc olympique relève de la disponibilité de ressources financières nécessaires pour mettre à niveau les bâtiments/infrastructures mais aussi, nécessaires pour améliorer l'accessibilité à ces derniers pour les clientèles moins fortunées.

Outre l'aspect financier qui domine l'ensemble des préoccupations des intervenants, les relations entre les intervenants présents sur le site ou concernés par le site et les modalités de gestion des activités du Parc olympique en général semblent poser de graves problèmes. Rassemblées sous le terme de "gouvernance ", ces préoccupations relèvent principalement de la gestion générale du site, des lacunes en matière de concertation entre les propriétaires ou parties prenantes. L'idée générale qui ressort de ces préoccupations est l'inadéquation des structures actuelles pour harmoniser et faire émerger une vision forte pour l'ensemble du Parc olympique. Du point de vue des intervenants consultés, seule une concertation accrue souple mais efficace ressort comme condition de valorisation du site dans son ensemble. Pour « l'environnement » ou « l'écosystème » sportif comme tel, ce besoin de concertation et d'harmonisation entre les différents acteurs des différents créneaux sportifs semble aussi être une condition sine qua non d'une consolidation de la vocation sportive du Parc olympique qui puisse faire consensus et rallier autant les intervenants du champ sportif que ceux des autres secteurs concernés par l'avenir de ce territoire. Malgré l'omniprésence des préoccupations sur ce thème, il n'y a pas de solution concrète et commune qui émerge de la part des intervenants sinon, à l'occasion, l'idée générale d'une redéfinition de la mission et du fonctionnement de la RIO.

À ce titre, plusieurs nous ont indiqués que des mesures précises pourraient être prises pour améliorer cette situation. Un membre d'une organisation sportive provinciale indique que : "Au-delà de la mise en place d'un plan d'action concerté, la RIO devrait organiser des ateliers de discussion avec les Fédérations sportives de façon à connaître et éventuellement intégrer certains de leurs projets et initia- 
tives événementielles. » Pour un représentant d'une Fédération sportive provinciale :

«Une structure de concertation plus flexible devrait être mise en place. Celle-ci pourrait notamment prendre la forme de tables de concertation thématiques (sport d'élite, sport étudiant, sport pour les personnes vivant avec une déficience, etc.) et récurrentes (organisées 1 à 2 fois par année) où diverses organisations présentes ou non sur le site du Parc olympique pourraient participer dépendamment de leur intérêt par rapport à la problématique abordée. »

Toujours au sujet de ces logiques de gouvernance, différentes personnes questionnées indiquent que le secteur privé a sa place sur le Parc olympique, à la fois via des activités et des services permettant d'animer et de dynamiser ce territoire mais aussi pour augmenter la rentabilité globale des actifs sur le site. Sur ce point, il y a une grande ouverture pour certains à permettre une commandite qui renommerait plusieurs éléments (certains espaces ou bâtiments, le stade lui-même, etc.) mais globalement les acteurs veulent maintenir le label « olympique » à l'ensemble du site.

Par ailleurs, certains acteurs interviewés reprochent à la RIO de s'accaparer trop souvent, dans les médias et à travers divers supports promotionnels, l'organisation de certains championnats et autres compétitions sportives programmés sur le site du Parc olympique mais organisés par diverses Fédérations. Pour ces dernières, cette reconnaissance médiatique et de ce fait cette visibilité devraient être plus équitables. Pour un acteur gérant certains espaces sportifs du Parc olympique : «Ces ententes promotionnelles déficientes brisent le lien de confiance avec la RIO et freinent la mise en place d'une gouvernance saine et respectueuse. »

Finalement et de façon unanime, tous les intervenants rencontrés ont déclaré que la question du toit du stade était la première priorité et la condition de base de la mise en valeur de la vocation sportive du Parc olympique. La problématique actuelle empêche l'utilisation du stade - plus précisément de sa surface principale - pendant quelques mois à chaque année ce qui diminue sa potentielle utilisation, affaiblit son image de marque et entretient l'aura négative autour du bâtiment et du site en général. Parallèlement et de nouveau pour tous les interve- nants consultés, il y a une mise à niveau importante à réaliser de certains bâtiments et espaces du site. Le stade bien sûr, au-delà de la question du toit, mérite d'être revampé tant à l'intérieur qu'à l'extérieur. Ainsi, des investissements majeurs sont nécessaires pour moderniser, adapter et améliorer l'état de l'équipement. Ces travaux concernent autant la structure et l'architecture elle-même, que les services et les composantes internes au stade.

\section{Le Parc olympique : objet com- MERCIAL VS PARC URBAIN OUVERT SUR LES POPULATIONS LOCALES}

Les résultats issus des entrevues semi-dirigées ainsi que l'analyse des diverses sources documentaires mettent en lumière certaines divergences au niveau du développement de la vocation sportive du Parc olympique de Montréal. En effet, le débat sur les sources de financement indispensables pour mettre à niveau le stade et l'ensemble du Parc olympique et le renouvellement des stratégies de gouvernance corrélées polarise les intervenants interviewés sur deux visions de développement territorial mises fréquemment en opposition.

Une première vision s'articule autour d'une présence accrue de partenaires du secteur privé tant dans la commandite, la gestion, la location que dans la propriété de certains éléments du Parc olympique. Une présence qui pourrait prendre plusieurs formes au point de voir certains symboles forts (le stade, certains espaces ou bâtiments, l'ensemble du site luimême) être complètement renommés avec le nom des entreprises ou des institutions concernées. De même, plusieurs établissements commerciaux pourraient s'installer à divers endroits sur le site ou dans certains bâtiments et structures existantes afin de répondre au besoin de services d'appoint (restauration, détente, hébergement, etc.) et d'animation, des services qui ont beaucoup été demandés à travers les rapports déposés. Ces commerces pourraient par ailleurs garder une « saveur » sportive avec des bars sportifs, des détaillants d'articles de sport et autres commerces de sport spécialisés dans la vente et l'animation. Poussée à son extrême limite, cette vision où les commerces, le secteur privé et le divertissement seraient visibles au maximum, avec une nette volonté d'encourager une rétention optimale des visiteurs et clients par une animation continue et une multitude 
de services et d'aménités, cette vision pourrait être comparée à un grand parc d'amusement commercial à thématique sportive. Le sport d'élite, les sports amateurs et les besoins locaux en sport y auraient leur place mais assujettie à un impératif de rentabilité et de commercialisation.

La deuxième vision privilégie surtout un financement public, un financement qui serait justifié par la dimension publique et citoyenne de l'héritage olympique, justifié aussi par le besoin de « redonner » le Parc olympique à la population en assurant une accessibilité optimale à ses équipements et services. Ainsi, le site et les principaux bâtiments pourraient conserver leur nom original (surtout, ne pas toucher au label « olympique »...). Le sport amateur, les fédérations sportives et de loisir, les établissements scolaires ou autres pourraient enfin se prévaloir d'un accès à certains espaces auparavant inaccessibles. Les aménagements physiques sur le site seraient plus respectueux de la symbolique olympique et permettraient la création d'un véritable " parc » public tourné vers les besoins de la population (détente, loisirs, sports, plein air). Le verdissement du site serait ainsi vraisemblablement plus important au point de créer plusieurs espaces de repos, de détente et de déambulation. Le Parc olympique deviendrait ainsi un grand parc urbain public multifonctionnel mais à dominante sportive. Pour plusieurs, la justification de fonds publics importants pour une telle vision doit passer par une valorisation accrue du sport et des saines habitudes de vie dans la société québécoise, un investissement collectif ayant des bénéfices réels sur les coûts de santé de l'ensemble de la collectivité et sur la qualité de vie en général.

Ces deux visions antagonistes rejoignent certains des modèles de gouvernance énoncés par Poynter (2009). Alors que la seconde vision présentée s'apparente assez aisément à la structure de gestion entièrement publique définie par cet auteur, la première vision est par contre beaucoup plus difficile à catégoriser. Elle semble se fonder sur un modèle partenarial où le secteur privé serait dominant, mais en même temps laisse de la place à des acteurs de la société civile. Au-delà de cette relative complexité à modéliser ces deux conceptions de développement du Parc olympique de Montréal, il est également intéressant de noter que les différents discours obtenus via les entrevues menées font état d'un élément commun. En effet, pour l'ensemble des acteurs interviewés le redéveloppement de ce site doit viser un certain équilibre entre une rentabilisation financière pérenne et une acceptabilité sociale des décisions de gestion effectuées. Ces éléments rejoignent en partie les propos de Orueta et Fainstein (2008) ou encore de Davies (2002), où l'intégration de visées économiques néo-libéralistes dans les objectifs de régénération de tels sites engendre divers risques, dont celui d'accentuer encore davantage les inégalités sociales vis-à-vis de l'accessibilité de ces espaces ludiques.

Nos résultats démontrent également que le Parc olympique de Montréal semble être arrivé à un tournant de son histoire. En effet, la dette olympique remboursée, l'obligation de refaire le toit et les implantations récentes de diverses infrastructures sportives et culturelles d'importance sur le site conduisent de nombreux acteurs à indiquer qu'il est désormais temps de régénérer ces héritages via un plan de développement à long terme. Ces discours font donc référence, selon nous, à une régénération imposée, soit une régénération qui fait suite à une importante dégradation sur une longue période d'un territoire donné (PAP, 2004). Parallèlement, l'analyse fine des entrevues effectuées démontre que le processus de régénération de ce site doit passer par une analyse exhaustive des contraintes et des potentialités de ces espaces dans l'optique de mettre en place des stratégies de redéveloppement territorial adaptées, équilibrées et fondées sur les réalités urbaines et sociales locales. Comme l'expose Tallon (2010) dans ses travaux, la mise en place d'un tel processus de régénération urbaine est susceptible d'assurer un certain consensus de la part des populations résidentes et des médias, et parallèlement de permettre de prendre en compte d'éventuelles actions spéculatives et autres changements d'état et de statut de la zone régénérer.

Notre recherche révèle donc que dans le cas qui nous intéresse la régénération urbaine est de fait territorialisée puisqu'elle tend à limiter les disparités spatiales en renforçant les éléments homogènes constitutifs. Pour plusieurs acteurs interviewés, elle semble être intégrée dans les différentes pratiques et visions de l'aménagement urbain moderne. Elle s'inscrit de fait dans différentes temporalités : le temps présent pour la demande sociale, le temps futur à long terme pour les questions de durabilité 
et le temps passé pour la mise en valeur des héritages patrimoniaux. Si l'on se réfère aux travaux de Smith (2012), les objectifs de développement du Parc olympique de Montréal, distingués via cette étude, sont de fait multi variés en raison des notions de négociation, de hiérarchisation des actions, d'intégration de divers acteurs publics et privés et d'interrelation des stratégies visées. Parallèlement, le fait que plusieurs de nos résultats font ressortir que le redéveloppement de ce site doit être adapté aux besoins de différentes clientèles et aux exigences d'accueil d'événements variés renvoie selon nous à certaines conclusions de recherche de Pitts et Liao (2009). En effet pour ces auteurs, la mise en place de stratégies de régénération d'un site olympique comme celui de Montréal est conditionnée par la taille de la ville hôte, le rang de cette dernière dans la hiérarchie urbaine nationale voire continentale, et par conséquent par le pouvoir d'attraction de cette métropole auprès de divers investisseurs.

Finalement, nos résultats nous permettent de distinguer certains facteurs clés, selon les acteurs interviewés, susceptibles de garantir une cohésion de ce projet de redéveloppement territorial du Parc olympique de Montréal, et par le fait même d'obtenir une légitimité sociale. Ces éléments rejoignent pour la plupart les interprétations présentées par Smith (2012), Pitts et Liao (2009), Majoor (2008) et dans le PAP (2004). Ainsi la régénération de ce site semble devoir passer par une vision stratégique de développement à long terme fondée sur une volonté politique claire et un engagement sociétal affirmé et qui passe par une participation accrue de plusieurs acteurs et groupes d'intérêts issus de différents milieux. Parallèlement, il apparaît également nécessaire d'instaurer un système de contrôle et d'évaluation susceptible d'assurer une certaine flexibilité à ces opérations urbaines. Ce dernier élément rejoint spécifiquement les propos de Pitts et Liao (2009) et du PAP (2004) pour lesquels la démarche, dite de "monitoring", est généralement utilisée pour décrire les conditions principales du territoire à régénérer et ainsi être capable de fixer des objectifs d'intervention cohérents et pertinents avec le milieu. Étroitement corrélée au "monitoring", l'évaluation permet d'estimer l'avancée du projet par rapport aux objectifs initiaux et de réorienter ou réajuster l'opération si nécessaire.
En conclusion, il nous est possible de mentionner que l'ensemble des activités sportives au Parc olympique de Montréal apparaît un peu méconnu par la population en général, malgré le fait qu'elles sont bien présentes, diversifiées et comportent un potentiel qui ne semble pas exploité à sa juste valeur. Les consultations publiques sur l'avenir de ce territoire et nos entrevues démontrent clairement que cette vocation doit être maintenue et renforcée en privilégiant le sport de haut niveau et d'excellence. Les autres créneaux sportifs (amateur, scolaire, etc.) y ont leur place mais devront vraisemblablement s'adapter aux priorités qu'imposera ce sport d'élite dans l'utilisation des espaces et des équipements de même que dans la programmation. Les deux principaux défis à surmonter pour assumer cette tâche relèvent de la justification et de l'origine des ressources financières importantes pour mener à bien la mise en valeur du site et de son milieu d'insertion mais aussi des modalités de gouvernance et de gestion permettant une réelle efficacité et une véritable harmonisation des actions qui en découlent. Sur ce dernier point, il y a unanimité pour tous les répondants consultés : relever ces deux défis permettra de convaincre que ces investissements répondent à de réels besoins, que les potentiels méritent d'être exploités et que le site peut redevenir un objet de fierté collective.

Ainsi le phasage de l'ensemble de la stratégie de mise en valeur du Parc olympique est une dimension qui devra être étudiée avec soin dans un éventuel plan d'affaire. Les grandes opérations urbanistiques, incluant notamment un volet sportif et ludique, ont su progressivement s'imposer grâce à une vision globale et intégrée faisant véritablement consensus auprès de la population locale et des médias. Les stratégies sous-jacentes à ces opérations ont misé sur un long processus de planification, exhaustif et à l'écoute des besoins d'une grande diversité d'acteurs. Que ce soit pour le redéveloppement d'un quartier, le réaménagement d'un grand parc urbain ou encore la reconfiguration d'un complexe sportif, toutes ces opérations ont rassemblé les mêmes ingrédients : consultations publiques et concertation entre les acteurs, efforts pour informer et sensibiliser la population et les médias, prise en compte d'objectifs de développement durable et éco-responsable, «branding » et création d'une signature territoriale distinctive et respect de diverses formes d'intégration 
architecturale et urbanistique. À cette étape cruciale de l'histoire du Parc olympique, il semble temps d'investir et de préparer le long terme. Le défi est à la portée d'une véritable volonté politique d'investir et de refonder un sentiment de fierté collective pour l'ensemble du site et de ses équipements. Un grand projet urbain intégré, unique et innovateur, avec la renaissance d'un grand stade en son centre, pourrait véritablement devenir le projet catalyseur du Montréal festif et sportif de demain.

\section{Bibliographie}

Comité-conseil sur l'avenir du Parc Olympique, 2011. Rapport de synthèse sur les consultations publiques menées sur l'avenir du Parc Olympique de Montréal, Comité-conseil sur l'avenir du Parc Olympique, Montréal, 101 p.

DARGAn L., 2009. Participation and local urban regeneration : The case of the New Deal for communities in the UK, Regional Studies, vol. 43, n² 2, p. 305-317.

Davies J., 2002. The governance of urban regeneration : A critique of the governing without government thesis, Public Administration, vol. 80, $\mathrm{n}^{\circ}$ 2, p. 301-322.

Flyvbjerg B., Bruzelius N., Rothengatter W., 2003. Megaprojects and Risk. An Anatomy of Ambition, Cambridge University Press, Cambridge, 207 p.

Gibbons A., Wolff N., 2012. Introduction : Re-writing London and the Olympic City : Critical implications of "Faster, Higher, Stronger", City, vol. 16, n 4, p. 439-445.

GlynN M.A., 2008. Configuring the field of play: How hosting the Olympic Games impacts civic community, Journal of Management Studies, vol. 45, n 6, p. 1117-1146.

Gold J., Gold M. (dir.), 2011. Olympic cities : City agendas, planning and the World's Games, 1896-2016, Routledge, Londres, $444 \mathrm{p}$.

Hay A., Cashman R. (dir.), 2008. Connecting Cities: Mega Event Cities, SOPA Publishers, Sydney, 172p.

Lascoumes P., Le Galès P., 2007. Introduction: Understanding Public Policy through Its Instruments - From the Nature of Instruments to the Sociology of Public Policy Instrumentation, Governance, vol. 20, n 1, p. 1-21.

Latouche D., 2011. Montreal 1976, in Gold J., Gold M. (dir.), Olympic cities: City agendas, planning and the World's Games, 1896-2016, Routledge, Londres, p. 247-267.

Liao H., PitTs A., 2006. A brief historical review of Olympic urbanism. International Journal of the History of Sport, vol. $23, n^{\circ} 7$, p. 1232-1252.
Majoor S., 2008. Progressive planning ideals in a neo-liberal context, the case of Orestad Copenhagen, International Planning Studies, vol. 13, n², p. 101-117.

Mévellec A., 2006. Gouverner par les instruments, Revue Canadienne de Science Politique, vol. 39, n² 2, p. 445-446.

Orueta F., Fainstein S., 2008. The new mega-projects : Genesis and impacts, International Journal of Urban and Regional Research, vol. 32, p. 759-767.

Programme d’Actions Prioritaires (PAP), 2004. Lignes directrices pour une régénération urbaine dans la région méditerranéenne, Centre d'Archives Régionales, Split, 53 p.

Pitts A., Liao H., 2009. Sustainable olympic design and urban development, Routledge, Londres, 238p.

Poynter G., 2009. Literature review : Olympic legacy governance arrangements. Rapport pour la London Assembly, LERC/ UEL, Londres, 47 p.

Raco M., 2005. A step changeor a step back: The Thames Gateway and the re-birth of the urban development corporations, Local Economy, vol. 20, n² 2, p. 141-153.

Roult R., Lefebvre S., 2010. Planning and reconversion of olympic heritages: The Montreal Olympic stadium, International Journal of the History of Sport, vol. 27, $\mathrm{n}^{\circ}$ 16-18, p. 2731-2747.

Shirai H., 2009. From Global field to Local neighbourhood. Sustainable transformation of the Olympic park for the City, The London School of Economics and Political Science, Londres, $115 \mathrm{p}$.

SILk M., 2002. "Banga Malaysia": Global sport, the city and the mediated refurbishment of local identities, Media, Culture and Society, vol. 24, p. 775-794.

Sмiтн A., 2012. Events and urban regeneration. The strategic use of events to revitalise cities, Routledge, Londres, 302 p.

Tallon A., 2010. Urban Regeneration in the UK, Routledge, Londres, $322 \mathrm{p}$.

Thévoz L., 2013. Construire la gouvernance territoriale : les principales dimensions d'un processus de concertation, multi acteur et participatif, in Vodoz L., Thévoz, L., Faure P. (dir.). 2013. Les horizons de la gouvernance territoriale, Presses polytechniques et universitaires romandes, Lausanne, p. 231-242.

Vodoz L., Thévoz L., Faure P. (dir.), 2013. Les horizons de la gouvernance territoriale, Presses polytechniques et universitaires romandes, Lausanne, $260 \mathrm{p}$.

Westerbeek H.M., 2009. The Amsterdam Olympic Games of 1928 and 2028: will city heritage inform legacy intent?, Sport in Society, vol. 12, n 6, p. 776-791.

Zhang J., Wu F., 2008. Mega-event marketing and urban growth coalitions, Town Planning Review, vol. 79, $\mathrm{n}^{\circ}$ 2-3, p. 209-226. 